\title{
Non-ST-elevation myocardial infarction complicating carcinoid syndrome
}

\author{
Ayman Elbadawi ${ }^{1}$, Erfan Alotaki ${ }^{1}$, Islam Y. Elgendy ${ }^{2}$, Hend Shahin ${ }^{3}$ and Jeremiah Depta ${ }^{4}$ \\ ${ }^{1}$ Department of Internal medicine, Rochester General Hospital, Rochester, New York, USA \\ ${ }^{2}$ Department of Cardiology, University of Florida, Florida, USA \\ ${ }^{3}$ Pharmaceutical Department, Futures University, Cairo, Egypt \\ ${ }^{4}$ Department of Cardiology, Rochester General Hospital, Rochester, New York, USA
}

\section{Introduction}

Neuroendocrine tumors (NETs) are a heterogeneous group of neoplasms associated with active secretion of vasoactive substances leading to carcinoid syndrome. We present a rare case of poorly differentiated NET presenting with Non-ST-elevation myocardial infarction (NSTEMI).

\section{Case report}

A 72 year-old-female presented with intermittent chest pain at rest and generalized fatigue for 3 days. Patient had a medical history significant for diabetes mellitus, chronic kidney disease and was recently diagnosed with poorly differentiated NET of an unknown primary site. On presentation, she had a heart rate of 105 beats per minute, a blood pressure of $137 / 87 \mathrm{mmHg}$, a respiratory rate of 18 breaths/ min, a temperature of $98.2 \mathrm{~F}$ and oxygen saturation level of $94 \%$ on room air. Lung examination showed normal breathing sounds. Cardiac examination showed rapid and regular heart beats, with normal heart sounds and no audible murmur. She had no jugular venous distention or peripheral edema. The rest of the exam was unremarkable. Chest $\mathrm{X}$-ray was normal. Electrocardiogram showed normal axis, sinus tachycardia with associated 1-mm ST-segment depression in inferolateral leads (Figure 1). Transthoracic echocardiogram revealed normal left ventricular ejection fraction (60\%), with segmental wall motion abnormality involving basal and mid inferior wall, normal valvular morphology and function as well as normal right ventricle size and function. Computerized tomography angiogram of the chest didn't show any evidence of pulmonary embolism.

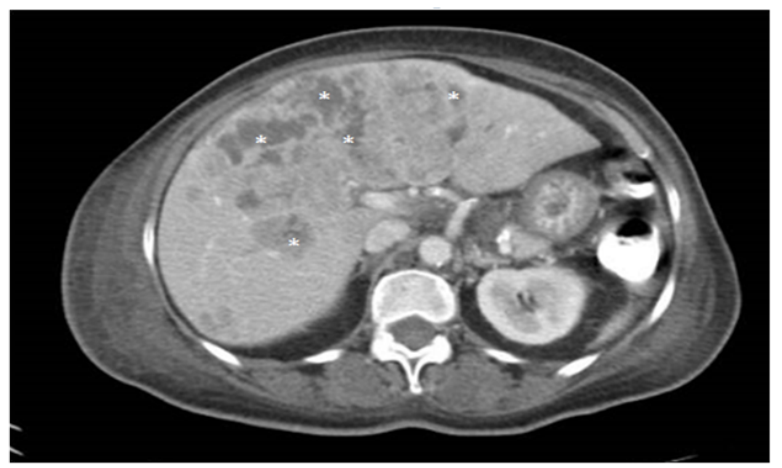

Figure 1. Electrocardiogram showing sinus tachycardia with associated 1-mm ST-segment depression in infero-lateral leads.
Pertinent laboratory studies revealed hemoglobin of $12 \mathrm{~g} / \mathrm{dl}$, white blood cell count of $14 \times 10^{3}$ cells/ull, procalcitonin $<0.05$ (normal $<0.1$ ), serum creatinine of $1.2 \mathrm{mg} / \mathrm{dl}$, elevated troponin level which peaked at $8.04 \mathrm{ng} / \mathrm{ml}$, serum serotonin of $1280 \mathrm{ng} / \mathrm{ml}$, chromogranin A of $114500 \mathrm{ng} / \mathrm{ml}$ and 24 hours urinary 5-HIAA of $332 \mathrm{mg}$. Owing to poor prognosis and poor general condition, decision was made to conservatively mange her NSTEMI. She was started on a somatostatin analogue and a beta-blocker, with secondary improvement in her symptoms.

\section{Discussion}

NETs are heterogeneous group of tumors which vary in their histological and biological characteristics. Several classification systems have been proposed for NETs, but broadly they can be categorized into poorly differentiated NETs and well differentiated NETs [1]. Many of the NETs are capable of secreting various humoral factors producing constellation of symptoms; what is known as carcinoid syndrome [1]. Carcinoid syndrome is typically described in well differentiated neuroendocrine tumors of the digestive tract and lungs, however, occasionally NETs in other sites can cause carcinoid syndrome [1]. These tumor products are typically broken down in the liver, that's why carcinoid syndrome mostly occurs in cases of metastasis to the liver with secretion of tumor products into the systemic circulation. As many as 40 secretory products have been identified to contribute to carcinoid syndrome, most prominently are serotonin, histamine, kallikrein and prostaglandins [2].

Cardiac involvement in carcinoid syndrome occurs in as many as $50 \%$ patients, with approximately $20 \%$ of patients having cardiac symptoms as initial presentation [3]. Cardiac involvement is a major cause of morbidity and mortality in patients with carcinoid syndrome [3]. Right sided heart lesions are the predominant form of cardiac involvement, with fibrous deposits affecting valves and endocardium of right side of the heart [3]. The inactivation of the humoral substances

Correspondence to: Ayman Elbadawi, Department of Medicine, Rochester general hospital, 1425 Portland Avenue, Rochester, Tel: +1 (585) 943-7821; Fax: +1(585)922-4440; E-mail: Ayman.Elbadawi@rochesterregional.org

Key words: Neuroendocrine tumor, carcinoid tumor, non ST-elevation myocardial infarction

Received: March 04, 2017; Accepted: March 22, 2017; Published: March 25, 2017 
by the lung, generally protect left side of the heart from being affected. However, left sided heart lesions occur in less than $10 \%$ of cases of carcinoid syndrome mostly in setting of primary bronchial tumors or advanced tumors with extremely high levels of vasoactive substance [3]. NSTEMI complicating carcinoid syndrome has been rarely described in literature. Few case reports exist on vasospastic angina occurring in carcinoid syndrome and usually in patients with preexisting ischemic heart disease $[4,5]$. The mechanism for NSTEMI in our patient could be related to the extremely high serotonin levels. Previous studies have suggested that serotonin plays a role in coronary artery disease [6]. Serotonin has a diverse physiological effect on the cardiovascular system [7]. Serotonin has a vasodilator effect on blood vessels mediated by 5-hydroxytryptamine-1 (5-HT1) receptors, as well as release of endothelial derived relaxation factors (EDRF) from healthy endothelium [7]. On the other hand, it has a vasoconstrictive effect mediated by direct action on 5-hydroxytryptamine-2 (5-HT2) receptors on endothelium and vascular smooth muscle cells, and indirectly by potentiating the effect of other vasoconstrictive agents such as histamine, angiotensin II, and norepinephrine. The vasoconstrictive effect is more prominent in atherosclerotic vessels owing to the lack of EDRF mediating vasodilatory effect of serotonin. The diverse vascular effect of serotonin could even cause different tonic responses in segments of the same coronary vessel [8]. Athero-thrombosis could have also contributed to the NSTEMI as a consequence of the hypercoagulable state associating malignancies. Also, experiments have shown that high serotonin levels may play a role in endothelial injury and augment the effects of other platelet activation agonists, both of which might lay the groundwork for thrombosis [6]. However, NETs are more typically associated with venous thrombosis [2] rather than arterial thrombosis.

In addition to the fore-mentioned mechanisms mediated by serotonin, other polypeptides and amines secreted by carcinoid syndrome could theoretically augment the coronary vasospastic response [5]. To summarize, we report a very rare presentation for carcinoid syndrome with NSTEMI. We assume this was secondary to the extremely high levels of serotonin in our patient's blood, with resulting vasospasm and possible athero-thrombosis. Cardiovascular complications such as myocardial infarction should be kept in consideration when managing cases of carcinoid syndrome, especially with very high levels of vasoactive substances.

\section{Conflicts of interest}

Authors have no conflicts of interest to declare.

\section{References}

1. Lawrence B, Gustafsson BI, Chan A, Svejda B, Kidd M, et al. (2011) The epidemiology of gastroenteropancreatic neuroendocrine tumors. Endocrinol Metab Clin North Am 40: 1-18. [crossref]

2. Chejfec G, Falkmer S, Askensten U, Grimelius L, Gould VE (1988) Neuroendocrine tumors of the gastrointestinal tract. Pathol Res Pract 183: 143-154. [crossref]

3. Pellikka PA, Tajik AJ, Khandheria BK, Seward JB, Callahan JA, et al. (1993) Carcinoid heart disease. Clinical and echocardiographic spectrum in 74 patients. Circulation 87: 1188-1196. [crossref]

4. Anselmi A, Corbineau H, Boulmier D, Ruggieri VG. (2013) Early diffuse coronary artery spasm after heart valve surgery in the carcinoid syndrome. Journal of cardiac surgery 28: 402-403.

5. Eapen DJ, Clements Jr S, Block P, Sperling L. (2012) Metastatic carcinoid disease inducing coronary vasospasm. Tex Heart Inst J 39: 76. [crossref]

6. Vikenes K, Farstad M, Nordrehaug JE (1999) Serotonin is associated with coronary artery disease and cardiac events. Circulation 100: 483-489. [crossref]

7. Frishman WH, Grewall P (2000) Serotonin and the heart. Ann Med 32: 195-209. [crossref]

8. Myers JH, Mecca TE, Webb RC. (1985) Direct and sensitizing effects of serotonin agonists and antagonists on vascular smooth muscle. Journal of cardiovascular pharmacology 7: 44-48.

Copyright: $₫ 2017$ Elbadawi A. This is an open-access article distributed under the terms of the Creative Commons Attribution License, which permits unrestricted use, distribution, and reproduction in any medium, provided the original author and source are credited. 